\title{
Pseudo-atomic Structure of Coxsackievirus A21 Complexed with Its Cellular Receptor, ICAM-1
}

C. Xiao ${ }^{*}$, C. M. Bator ${ }^{*}$, P. Chipman", T. S. Baker ${ }^{*}$, R. J. Kuhn ${ }^{*}$, E. Wimmer ${ }^{* *}$, A. Craig ${ }^{* * *}$, and M. G. Rossmann

* Department of Biological Sciences, Purdue University, West Lafayette, IN 47907-1392, USA

** Department of Molecular Genetics and Microbiology, School of Medicine, State University of New York, Stony Brook, NY 117945222, USA

Molecular Parasitology Group, Institute of Molecular Medicine, John Radcliffe Hospital, Oxford OX3 9DS, United Kingdom

Coxsackievirus A21 (CAV21), like human rhinoviruses (HRVs), is a causative agent of the common cold. CAV21 uses the same cellular receptor, intercellular adhesion molecule-1 (ICAM-1), as does the major group of HRVs. Moreover, unlike HRVs, CAV21 is stable over wide pH range. The CAV21 RNA genome has about $80 \%$ amino acid sequence identity to polioviruses (PVs), which utilize CD155 as their receptor. In contrast, CAV21 has only about 50\% identity to HRVs. Interaction between the virus and the receptor had been studied by fitting atomic structure into a $26 \AA$ resolution cryo-EM map. [1] However, the low resolution of the cryo-EM density limited the fitting accuracy and affected the interpretation of the interactions. Furthermore, the structure of CAV21, which had been fitted into the cryo-EM map, was modeled from the homologous structures of poliovirus 1 structure instead of using an X-ray crystallographic determined crystal structure.

The cryo-EM image reconstruction of CAV21 complexed with 5-domain soluble ICAM-1 has now been improved to about $11 \AA$ resolution. In addition, CAV21 has been crystallized at three different $\mathrm{pHs}$ and the structure has been solved to $3.5 \AA$ resolution. A computer program has been used to fit the atomic structures into the cryo-EM density map. Accurate structural information has been obtained from this pseudo-atomic structure of CAV21/ICAM-1 complex. Besides the wild type 5 domain ICAM-1, a naturally occurring single residue mutant of ICAM -1 (kili fi ICAM-1 [2]) was used also for cryo-EM studies.

The main binding site of ICAM-1 on the CAV21 is located at the southern rims of the canyon, which includes the "puff" of VP2, the GH loop of VP1 and the GH loop of VP3. Sequence comparisons between CAV21, PV and HRVs shows that the viral residues involved in binding the receptor differ among picornaviruses. The surface of ICAM-1 that binds to CAV21 consists primarily of one side of the domain 1. The receptor does not penetrate further down to the canyon su ggests that the frozen structure of the complex obtained by cryo -EM may be the initial intermediate prior to viral disassembly and cell entry.

References

[1] C. Xiao, et al., J. Virol. 75 (2000) 2444

[2] A. Craig, et al., Human Mol. Genetics 9 (2000) 525 

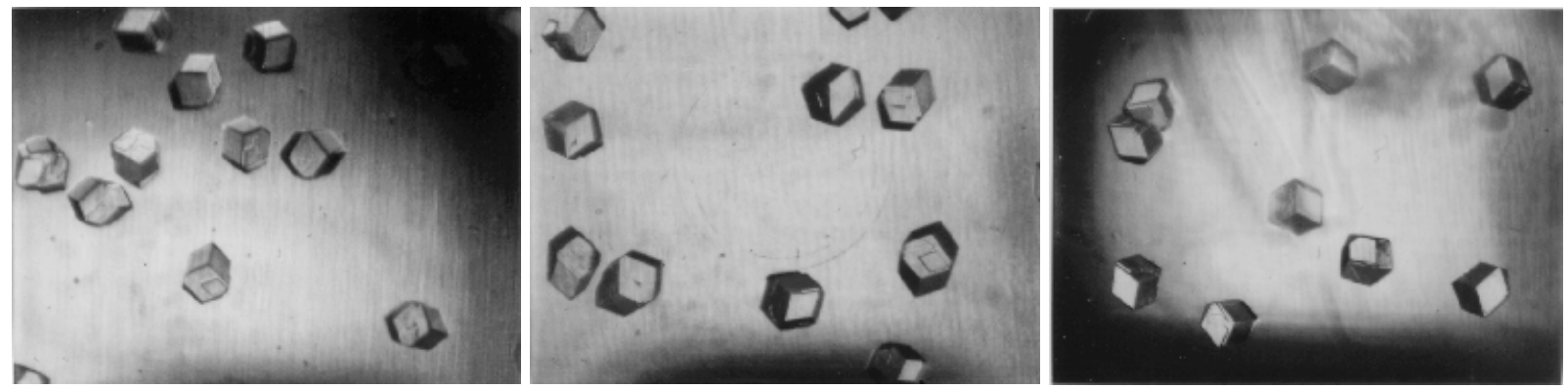

FIG 1 CAV21 crystals. From left to right, the crystals are grown in pH 5.5, 6.4, 7.2 respectively.

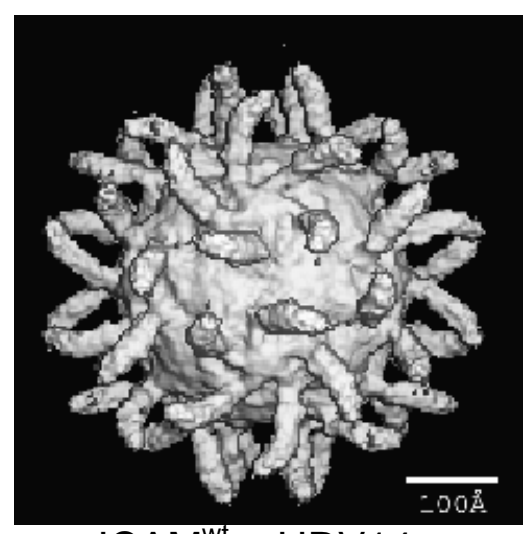

$\mathrm{ICAM}^{\mathrm{wt}}+\mathrm{HRV} 14$

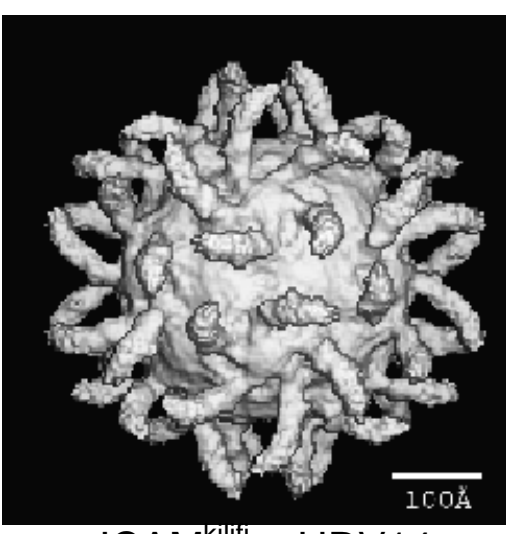

ICAM $^{\text {KIIIII }}+$ HRV14

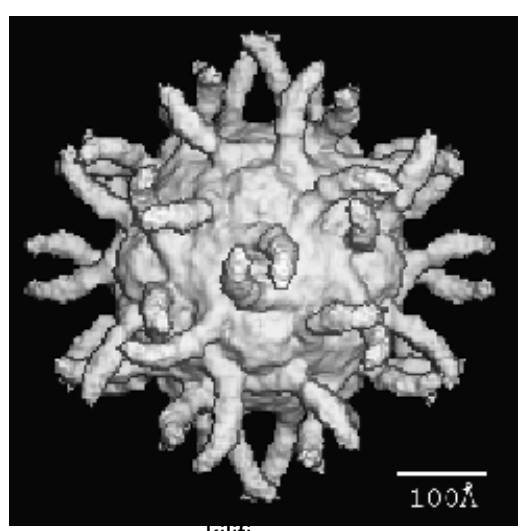

$\mathrm{ICAM}^{\mathrm{KIIIfl}}+\mathrm{CAV} 21$

FIG 2 cryo-EM reconstruction of ICAM with HRV14 and CAV21
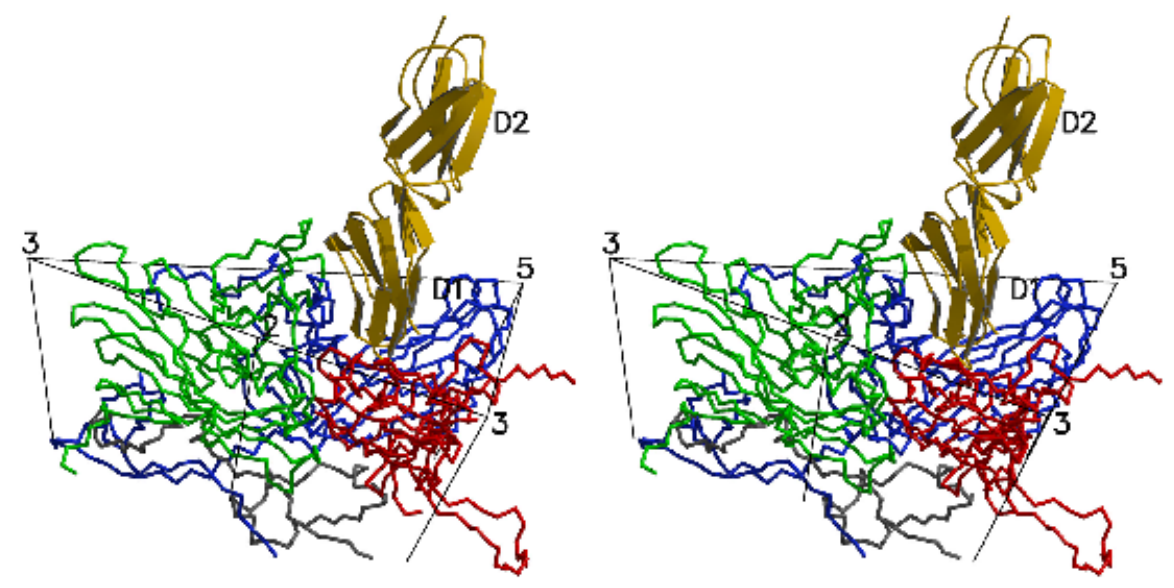

FIG 3 Stereo view of a ribbon diagram of the first two domains of ICAM-1 (yellow) bound to one icosahedral asymmetric unit of CAV21. Ribbons of VP1, VP2, VP3 and VP4 are shown in blue, green, red and grey respectively. 